B. Doll*, E. Calderón del Rivero, J. Hepp, T. Pickel, C. Buerhop, R. Knecht, C. Camus, J. Hauch, J. Parisi and C.J. Brabec

\title{
Quantitative Assessment of the Influence of Camera and Parameter Choice for Outdoor Electroluminescence Investigations of Silicon Photovoltaic Panels
}

https://doi.org/10.1515/zna-2019-0025

Received January 24, 2019; accepted May 27, 2019; previously published online June 27, 2019

\begin{abstract}
With the spread of photovoltaics (PV) and increasing diversity in PV panel technology, quantitative comparison of the modules is highly desirable for consistent on-site quality assessment. Electroluminescence imaging reveals many defects, such as macroscopic crystal or electrical contact defects, but quantitative comparison outside the laboratory without controlled environment is still difficult, especially for different detector technologies. Here, we show how this problem can be addressed by adding reference spots in the module area: One passive dark spot and an active bright spot
\end{abstract}

\footnotetext{
*Corresponding author: B. Doll, Friedrich-Alexander University Erlangen-Nürnberg, Faculty of Engineering, Materials for Electronics and Energy Technology, Erlangen, Germany; Graduate School in Advanced Optical Technologies, Erlangen, Germany; and Helmholtz-Institut Erlangen-Nürnberg, Erlangen, Germany, E-mail: bernd.doll@fau.de

E. Calderón del Rivero: SUNSET Energietechnik GmbH, Adelsdorf, Germany, E-mail: ecalderondelrivero@gmail.com

J. Hepp: Friedrich-Alexander University Erlangen-Nürnberg, Faculty of Engineering, Materials for Electronics and Energy Technology, Erlangen, Germany; Graduate School in Advanced Optical Technologies, Erlangen, Germany; and Helmholtz-Institut ErlangenNürnberg, Erlangen, Germany, E-mail: Johannes.Hepp@fau.de T. Pickel and C. Buerhop: Helmholtz-Institut Erlangen-Nürnberg, Erlangen, Germany, E-mail: t.pickel@fz-juelich.de (T. Pickel); c.buerhop-lutz@fz-juelich.de (C.Buerhop)

R. Knecht: Carl von Ossietzky University Oldenburg, Oldenburg, Germany, E-mail: robin.knecht@uni-oldenburg.de

C. Camus: LayTec AG, Berlin, Germany, E-mail: ccamus@aol.com
}

J. Hauch: Friedrich-Alexander University Erlangen-Nürnberg, Faculty of Engineering, Materials for Electronics and Energy Technology, Erlangen, Germany; and Helmholtz-Institut Erlangen-Nürnberg, Erlangen, Germany, E-mail: j.hauch@fz-juelich.de

J. Parisi: Carl von Ossietzky University of Oldenburg, Institute of Physics, Energy and Semiconductor Research Laboratory, Oldenburg, Germany, E-mail: juergen.parisi@uni-oldenburg.de C.J. Brabec: Friedrich-Alexander University Erlangen-Nürnberg, Faculty of Engineering, Materials for Electronics and Energy Technology, Erlangen, Germany; Bavarian Centre for Applied Energy Research, Erlangen, Germany; and Helmholtz-Institut ErlangenNürnberg, Erlangen, Germany, E-mail: christoph.brabec@fau.de composed of a high-power back-contacted silicon PV cell. Those reference spots are used to evaluate the module's electroluminescence signal under different environmental conditions and to establish comparable results. Additionally, the comparison of images acquired with different camera technology detectors, such as silicon and InGaAs, is realised for signal levels.

Keywords: Defect Detection; Electroluminescence; Outdoor; Photovoltaic; Quantitative Comparison.

\section{Introduction}

Photovoltaic (PV) modules are of great interest due to the clean and easy energy production. Each cell is composed of semiconductor materials, typically boron- and phosphorous-doped silicon (Si), forming a pn junction with an internal potential gradient [1]. By the interaction of incident light, photons with an energy higher than the band gap energy of silicon (1.11 eV at $T=293 \mathrm{~K}$ ) [2] excite electrons in the valence band to the conduction band. The internal potential gradient in the pn junction separates the generated charge carriers and hence generates an electric current at the metallic terminals [3]. Non-harvested excited electrons relax to their initial energy state; their surplus energy is released in non-radiant (heat) and radiant recombination (luminescence) [1].

Additionally, the PV cells can be used directly as light-emitting diodes (LEDs) with an external current. This electrically stimulated luminescence is called electroluminescence (EL), and Si solar cells (SSCs) typically yield external quantum efficiencies (EQEs) of lower than $1 \%$ for the luminescence peak at around $1150 \mathrm{~nm}$ [4].

The EL intensity depends on the amount of forward current applied to the PV panel, following the "electrical current - light intensity" relation studied by Fuyuki et al. [5] and the "reciprocity" relation demonstrated by Rau [6]. The EL signal indicates the relative material performance. Although a high signal hints toward an unimpaired device, point defects and cracks, as well as a real degradation in the PV module, lead to local drops in the luminescence intensity and may influence the intensity distribution of the EL image [7-9]. 
Today, the most common cameras used for EL signal detection are either Si detector-based ones with high spatial resolution but low sensitivity for the Si luminescence peak, resulting in high-resolution images after long integration times of up to $40 \mathrm{~s}$ or indium gallium arsenide (InGaAs)-based detectors with low spatial resolution but high sensitivity at the $\mathrm{Si}$ luminescence peak, resulting in low resolution and high intensity images after short integration times of down to $1 \mathrm{~ms}$.

Furthermore, many other parameters or hardware setups have an influence on the resulting images. Numerous image correction procedures were developed [10, 11], but quantitative comparison among different detector technologies is not yet sufficiently developed or standardised.

In this paper, we focus on the quantitative comparability of EL images mainly evaluated by histogram analysis for various environmental conditions and different camera technologies by a normalisation step in the image post processing. Additionally, the basic EL parameters are defined to achieve reproducible images.

\section{Methodology and Preparatory Experiments}

\subsection{Cameras}

The cameras used for image acquisition were two with a $\mathrm{Si}$ (Si_A and Si_B) and two with an InGaAs (In_C and In_D) detector. Their main parameters are shown in Table 1. Because of the low EQE of the silicon detectors between 1000 to $1300 \mathrm{~nm}$, significantly higher integration times are typically necessary. In contrast, the InGaAs detectors exhibit a high EQE in the near-infrared NIR region between 900 to $1600 \mathrm{~nm}$ for the In_C camera or even an extended InGaAs detector from 400 to $1600 \mathrm{~nm}$ for the In_D camera. For both detector types, the quantum efficiency is shown in Figure 1 in comparison with the EL spectra of an SSC.

\subsection{Indoor and Outdoor EL Image Setups}

Indoor EL images were taken inside a dark chamber with four different cameras, each of them with different integration times. The PV

Table 1: Main specifications of the cameras used: overall weight of camera including lenses, approximate prices, resolution in pixels, typical integration times $T_{\text {int }}$, and the A/D dynamic range of the pixel depth.

\begin{tabular}{llllll}
\hline Name & Weight & Price & Pixels & $\boldsymbol{T}_{\text {int }}$ & A/D range \\
\hline Si_A & $\sim 4 \mathrm{~kg}$ & $20,000 €$ & $3.2 \mathrm{M}$ & $40 \mathrm{~s}$ & $12 \mathrm{bit}$ \\
Si_B & $<1 \mathrm{~kg}$ & $1200 €$ & $18 \mathrm{M}$ & $2 \mathrm{~s}$ & $12 \mathrm{bit}$ \\
In_C & $\sim 5 \mathrm{~kg}$ & $80,000 €$ & $0.3 \mathrm{M}$ & $20 \mathrm{~ms}$ & $12 \mathrm{bit}$ \\
In_D & $<1 \mathrm{~kg}$ & $40,000 €$ & $640 \times 512$ & $80 \mathrm{~ms}$ & $14 \mathrm{bit}$ \\
\hline
\end{tabular}

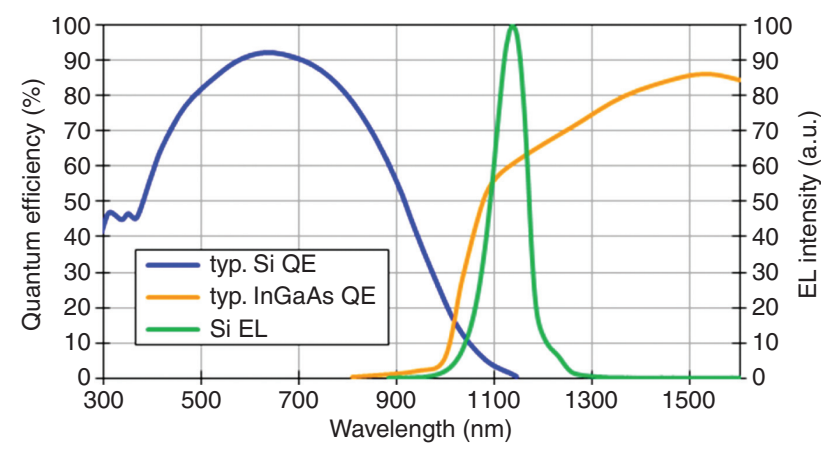

Figure 1: Quantum efficiencies of a typical Si and InGaAs detector in comparison with an EL spectra of a SSC [12].

panels were forward fed with $90 \%, 75 \%, 50 \%, 10 \%$, and $0 \%$ of their short circuit current $\left(I_{\mathrm{SC}}\right)$ at standard testing conditions (STCs). The integration times used differ for each camera and are mentioned separately in Section 3.2. PV panels were fixed in a vertical rack. Reference cell and black spot were set next to it. Cameras were mounted in an automated xyz stage focusing the PV panels perpendicularly. Power supplies and cameras are controlled from outside the dark chamber.

Outdoor EL images were taken at nighttime to avoid high noise signals from the sun. The PV panels were fixed to a $40^{\circ}$ tilted rack and forward fed with $90 \%$ of their respective $I_{\mathrm{SC}}$. Cameras were attached to a tripod and focusing the $\mathrm{PV}$ panel making an effective focus angle of $60^{\circ}$ between camera and PV panel.

\subsection{EL Signal Angle Dependency}

Additionally, in this study, the influence of the relative image acquisition angle on the images was studied. In Figure 2, the influence on the EL signal and resolution is shown. With the angle of tilt at $90^{\circ}$, the module is perpendicular to the camera view direction. The resolution follows the trigonometric functions and is sufficient for angles $>50^{\circ}$. On the other hand, the mean of the EL intensity signal dramatically drops for angles lower than $40^{\circ}$, mainly due to internal reflections in the glass layer of the module. Figure 3 shows the SSC module at a tilted angle of $40^{\circ}$ where the perspective distortion is still acceptable for image evaluation.

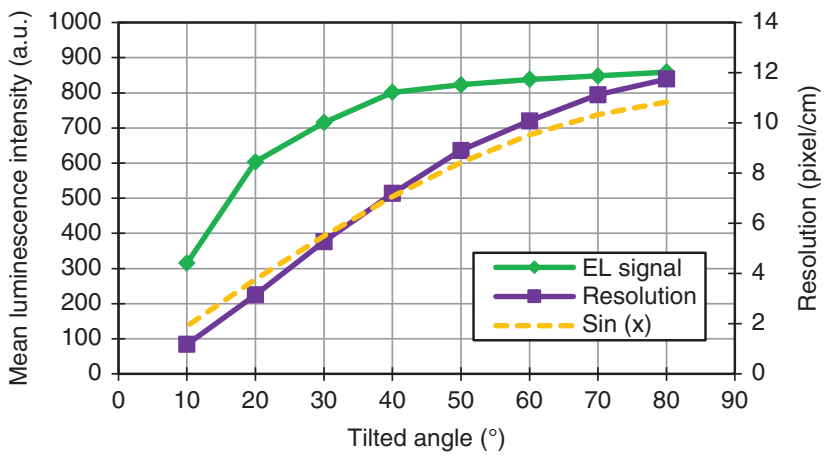

Figure 2: EL signal and resolution dependence on the tilted angle being defined as the angle between the SSC and camera view direction with the trigonometric sine function. 


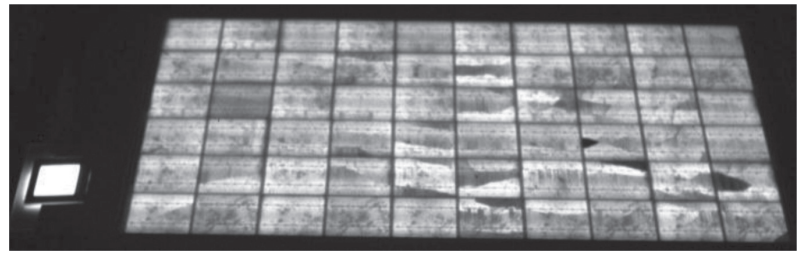

Figure 3: EL image at a tilted angle of $40^{\circ}$ taken with the Si_A camera with an integration time of $40 \mathrm{~s}$.

\subsection{Reference Cell Characterisation}

The goal to position a reference area in each EL image was realised with a high-power back-contacted monocrystalline 5" Si PV cell, with a peak power of $3.2 \mathrm{Wp}$. Additionally, the EL signal was homogenised with a plexiglas layer on top of the front surface. In Figure 4, the colourised EL image of the reference cell is shown. The mean value within the dashed rectangle was 3128 counts with a standard deviation of 188 counts under 6.4 A forward current. For further investigations, this dashed rectangle is called reference spot (RS).

Furthermore, the temperature was logged at the backside of the RS to determine the dependence of the EL signal to the cell temperature. The temperature was measured with an Almemo 2590 data logger, and the EL signal was averaged from the mean of the RS acquired with the In_C camera for 20 ms every 10 s. In Figure 5, the behaviour of the temperature influence on the EL signal for indoor and outdoor is shown. The rise of the EL signal is caused by a change of the non-radiant and radiant recombination probability and its temperature dependency for the indirect semiconductor material [13]. It clearly reveals that a minimum of 30 min warm-up thermalisation time is needed for precise and reproducible measurements.

\subsection{Normalisation Process}

The qualitative and quantitative comparison among the EL images is achievable by analysing them in the same intensity range and without external and internal influences. The recorded EL intensity $\left(I_{\mathrm{EL}}\right)$ detected by the cameras is shown with constant gain and ISO value

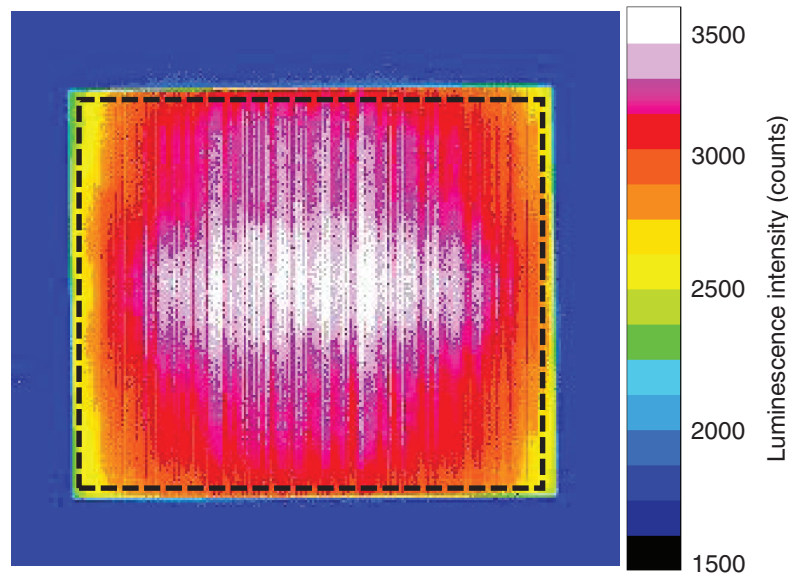

Figure 4: Homogenised EL reference cell image acquired with the In_C camera, with 6.4 A forward current and 20 ms integration time. The dashed rectangle indicates the area of interest. in (1), simplified from [14-16]:

$$
I_{\mathrm{EL}}=I_{\mathrm{S}}+I_{\mathrm{BG}, \mathrm{N}}+I_{\mathrm{DC}, \mathrm{N}}\left(T_{\mathrm{int}}, T\right)+I_{\mathrm{RO}, \mathrm{N}},
$$

where $I_{\mathrm{S}}$ is the pure signal intensity and the other intensities are additive noise intensities for background/stray light $I_{\mathrm{BG}, \mathrm{N}}$, and dark counts of the detectors $I_{\mathrm{DC}, \mathrm{N}}$ depending on the integration time $T_{\mathrm{int}}$ and temperature $T$ and the readout noise $I_{\mathrm{RO}, \mathrm{N}}$ depending on the camera. The background/stray light includes all types of radiation from the same direction as the SSC except from the SSC itself.

The signal intensity $I_{\mathrm{S}}$ depends on the variables in (2),

$$
I_{\mathrm{S}}=\frac{I_{\mathrm{C}} \cdot E Q E_{\mathrm{detector}} \cdot T_{\mathrm{int}} \cdot \tan \left(\Omega_{\mathrm{cam}, \mathrm{SC}}\right)}{d_{\mathrm{cam}, \mathrm{SC}} \cdot A \cdot f_{\mathrm{K}}}
$$

$I_{\mathrm{C}}$ is the SSC powering current, $\Omega_{\text {cam,SC }}$ and $d_{\text {cam,SC }}$ descript the angle and the distance between the camera and the SSC plane, $A$ is the aperture number, and $f_{\mathrm{K}}$ is a correction factor for material properties and behaviour of the SSCs.

For the quantitative comparison, all noise, operator-related, and geometric influences have to be eliminated. Therefore, a need exists to recalculate the luminescence intensities recorded with the different detector technologies. In order to achieve this recalculation, two spots in the SSC plane for each EL image are necessary. The black spot (BS), a metal plate covered with black plastic sheet, provides a mean intensity value $\overline{I_{\mathrm{BS}}}$ for subtraction of large area background noises. For the second one $\overline{I_{\mathrm{RS}, \mathrm{BS}}}$, the RS is used with its mean intensity value after the subtraction of the $\overline{I_{\mathrm{BS}}}$.

Finally, the normalisation process is conducted according to the following equation:

$$
I_{\mathrm{EL}, \mathrm{BS}}(x, y)=\sum_{i}\left[I_{\mathrm{EL}}\left(x_{i}, y_{i}\right)-\overline{I_{\mathrm{BS}}}\right] \cdot \frac{2000 \text { L.U. }}{\overline{I_{\mathrm{RS}, \mathrm{BS}}}}
$$

where the intensity values are normalised, between the values 0 and 2000 of the newly defined unit luminescence units (L.U.). Those values were chosen based on experience indicating that a depth of 2000 is sufficient for analysis of typical mono- or multicrystalline SSC. In Figure 6, the setup for the reference spots and PV modules is shown.

After the normalisation process, the influences to the external EL intensity are eliminated such that the normalised EL intensity is equal to signal intensity with the mean value $m$ between 0 and 2000 L.U.:

$$
I_{\mathrm{EL}}(0 \text { L.U. } \leq m \leq 2000 \text { L.U. })=I_{\mathrm{S}}
$$

\subsection{Slope Comparison and SNR}

The normalised images are analysed on the one hand by determining the slope of the normalised cumulative histogram and on the other hand by the signal to noise ratio (SNR) of the intensity values. For the slope analysis, the slope between the median and the $25 \%$-percentile provides a numeric value for image comparison. Furthermore, the SNR determines the ratio between useful EL signal and the noise in an image, see (5):

$$
\mathrm{SNR}=\frac{m_{\text {signal }}-m_{\text {noise }}}{\sigma_{\text {noise }}}
$$

where $m_{\text {signal }}$ is the mean value of a determined signal area and the $m_{\text {noise }}$ and $\sigma_{\text {noise }}$ are the mean value and the standard deviation of the BS area [15]. 

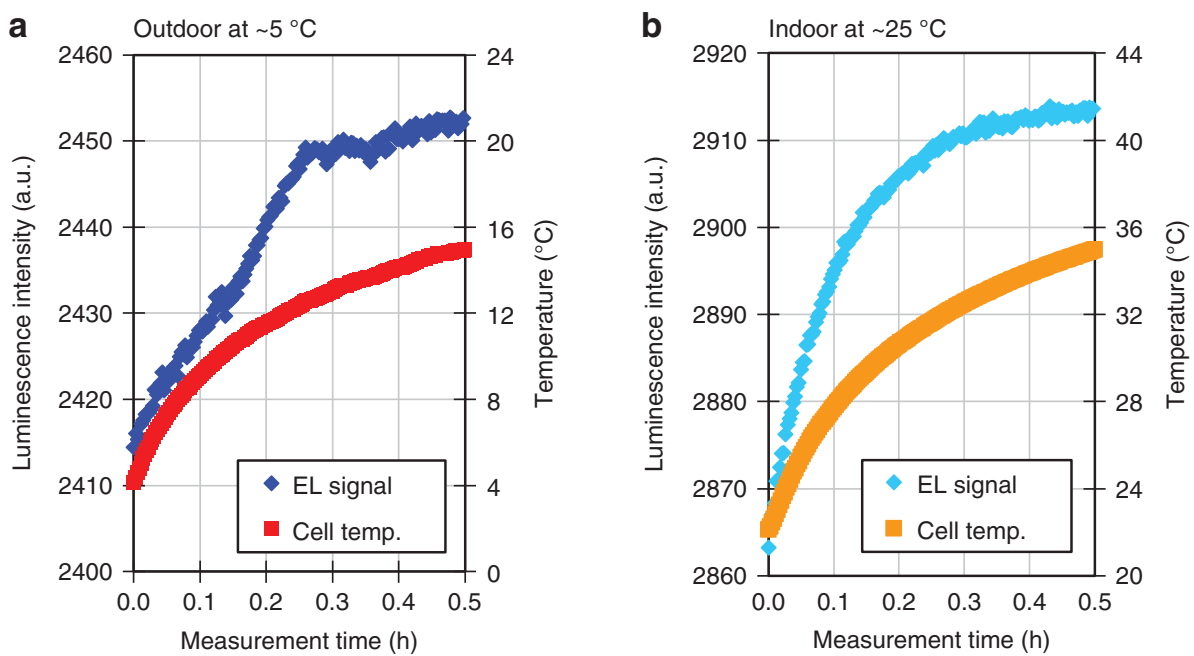

Figure 5: EL signal dependence from temperature change for (a) outdoor conditions at around $5{ }^{\circ} \mathrm{C}$ and (b) indoor conditions at around $25{ }^{\circ} \mathrm{C}$. The EL signal is measured with the luminescence intensity collected from the camera In_C for $20 \mathrm{~ms}$.

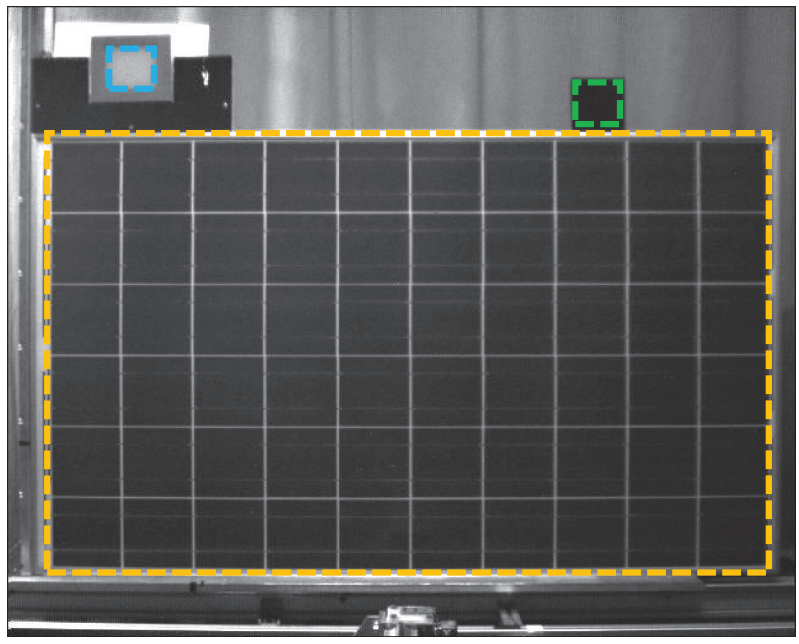

Figure 6: Setup of the reference spot (RS) marked blue, the black spot (BS) marked green, and the PV module marked yellow for indoor EL images.

\subsection{Comparability of Histograms}

Different camera systems or their different onboard image processing algorithms lack for quantitative image comparability for defect recognition.

Figure 7 gives a good example for the comparability problem. Camera Si_B and In_D show a high gain and ISO values. Those values could either be controlled or semi-automated. The In_C camera has a fixed gain, but due to manual non-uniformity correction, which was conducted before using, value comparability between different measurements is difficult. For the Si_A and In_C cameras, very steep slope values, which correspond to a lower intensity distribution range of the collected data, are observable. Thus, the EL signal intensity levels are harder to separate. The integration times of each camera were chosen to be as long as possible for the highest quality images, just limiting by saturation at the RS and the maximum

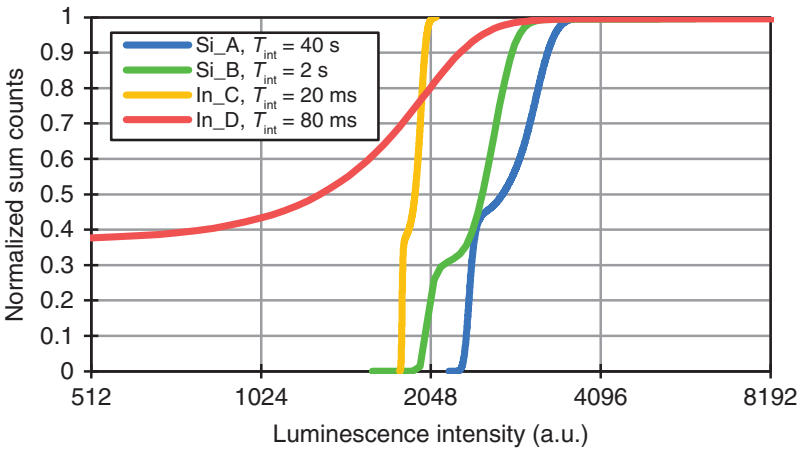

Figure 7: Comparison of the normalised cumulative histograms for typical indoor images of the four different cameras without normalisation with the corresponding integration times.

possible integration time. Other integration times are analysed in Section 3.2.

\section{Results and Discussion}

\subsection{Normalisation Process}

The comparability of all cameras can be increased by externally levelling the camera system with reference spots: One bright RS and one dark reference spot for postprocessing normalisation.

The normalisation process shifts the EL intensity captured from the cameras to a range of 0 to 2000 L.U. It does not modify the EL signal behaviour. In Figure 8, the normalised cumulative histograms after the normalisation process for all the cameras are shown. Compared with Figure 7, the histograms show similar behaviour and are in the same luminescence intensity range, except for the 


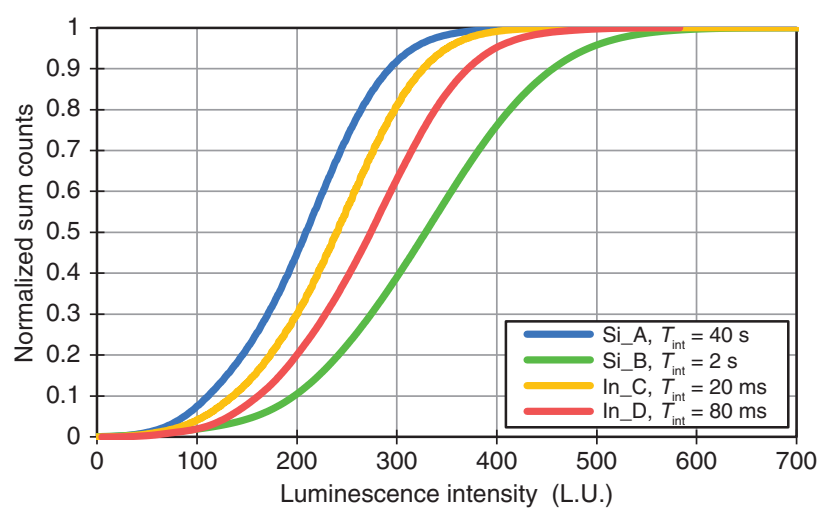

Figure 8: Comparison of the normalised cumulative histograms for the SSC module area in typical indoor images of the four different cameras after normalisation with the corresponding integration times.

Si_B camera. The explanation can be given due to the low linearity of the detector in the NIR region, which was not produced for low-light NIR imaging and therefore not optimised in this region.

\subsection{Comparability}

As an example, the quantitative comparability of the normalised cumulative histograms for a single camera is shown for a silicon detector Si_A in Figure 9 and for an InGaAs detector In_D in Figure 10. For each camera, EL images with different integration times $T_{\text {int }}$ and different currents were taken. All cameras showed similar behaviour. For higher integration times, the normalised cumulative histograms are nearly identical. But at low integration times of $10 \mathrm{~s}$ for Si_A and $20 \mathrm{~ms}$ for In_D, the slope is reduced, and the intensity level shifts to higher values. The reasons for those changes might be due either to camera internal increases of the readout noise influence

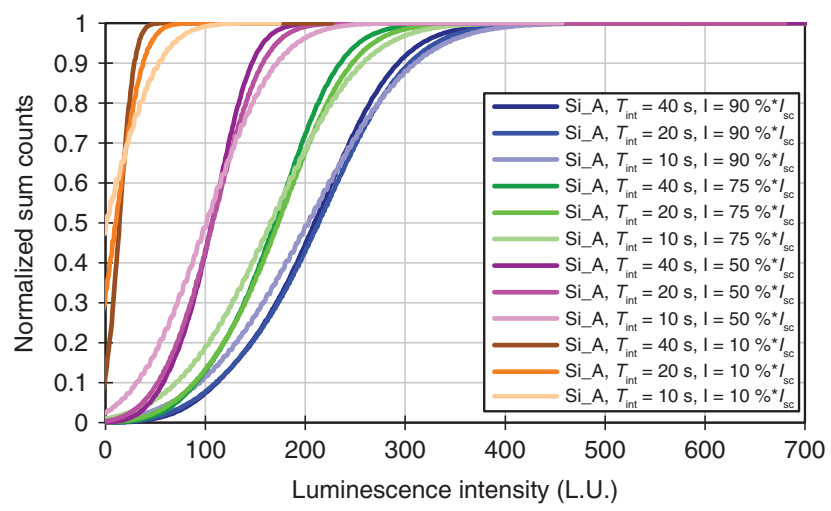

Figure 9: Normalised cumulative histograms for camera Si_A with different integration times $T_{\text {int }}$ and currents $/$ in percent of the module's $I_{\mathrm{sc}}$.

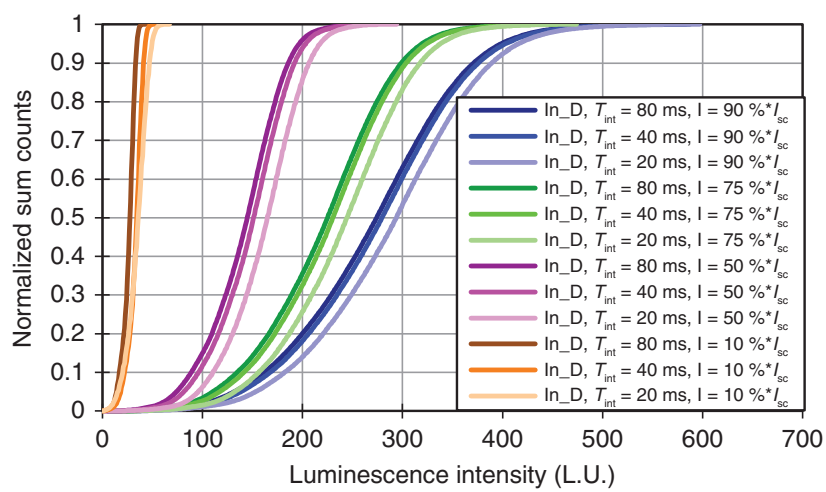

Figure 10: Normalised cumulative histogramms for camera In_D with different integration times $T_{\text {int }}$ and currents $/$ in percent of the module's $I_{\text {sc }}$.

for smaller integration times or to nonlinear image onboard processing like automated gain controls.

This clearly indicates the limit of the normalisation process for not properly chosen integration times.

Additionally, Figure 11 shows the slope values and the SNR of all cameras. High quality images will share a high SNR value and a low slope value. Higher noise levels for low integration times reduce the SNR levels for all cameras but with different factors. In our experience, SNR values higher than two to three are sufficient, which is a bit lower than the Rose criteria [17].

As already shown in Figures 9 and 10 with sufficiently high integration times and currents, the image quality and reproducibility of the normalisation procedure is high and robust. Nonetheless, the dashed linear fitting lines in Figure 11 clearly indicate that camera In_D is the most suitable for PV EL measurements, because the highest SNR and slope values are achieved. Cameras

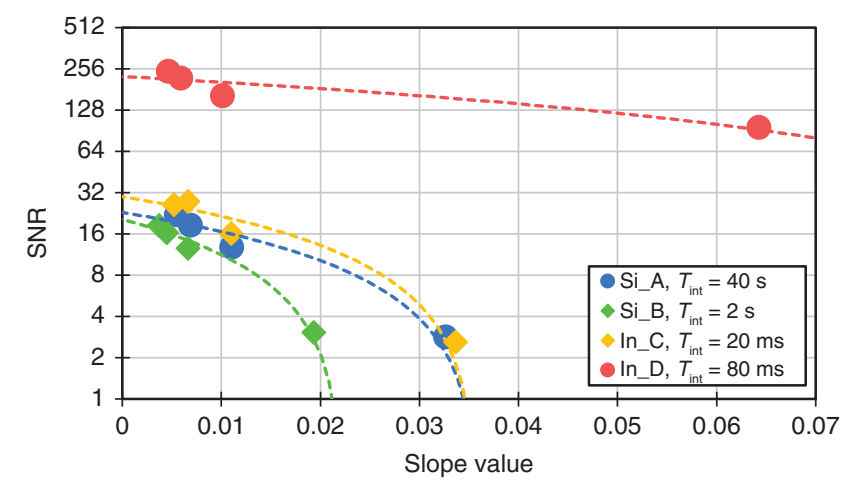

Figure 11: Quality analysis of the different cameras for the SNR and slope value of the EL cumulative histograms for different currents. The dashed lines indicate linear detector behaviour. The currents of $90 \%, 75 \%, 50 \%$, and $10 \%$ of the $I_{\text {SC }}$ starting with the lowest slope value and highest SNR on the left. In_D camera produces EL images of the highest quality, whereas camera Si_B shows the images with the lowest quality. 
Si_A and In_C show good images for laboratory cameras. As expected, the rebuild camera $\mathrm{Si}_{-} \mathrm{B}$ shows the lowest performance.

\subsection{Outdoor EL Imaging}

The main motivation to improve the quantitative comparison is the need to compare EL images taken in different environments. In this Section, we prove the robustness of the normalisation method for comparisons of indoor and outdoor EL images.

In Figure 12, both images are shown for camera In_C. Due to the tilt of the outdoor modules, the perspective changed for outdoor EL images. Figure 13 shows the normalised cumulative histograms of all the cameras with the slope values. The SNR in outdoor images suffers of a decrement of $60 \%$ to $40 \%$ in respect to the SNR in indoor images, stemming from higher noise levels in outdoor conditions. The normalisation does not eliminate the noise but reduces its intensity levels.

In order to perform a more accurate comparison between indoor and outdoor images, the acquisition angle was taken into count. Therefore, two images one from indoor and one from outdoor conditions, with the same tilt angle, forward current, and camera parameters, were compared (see Fig. 13).

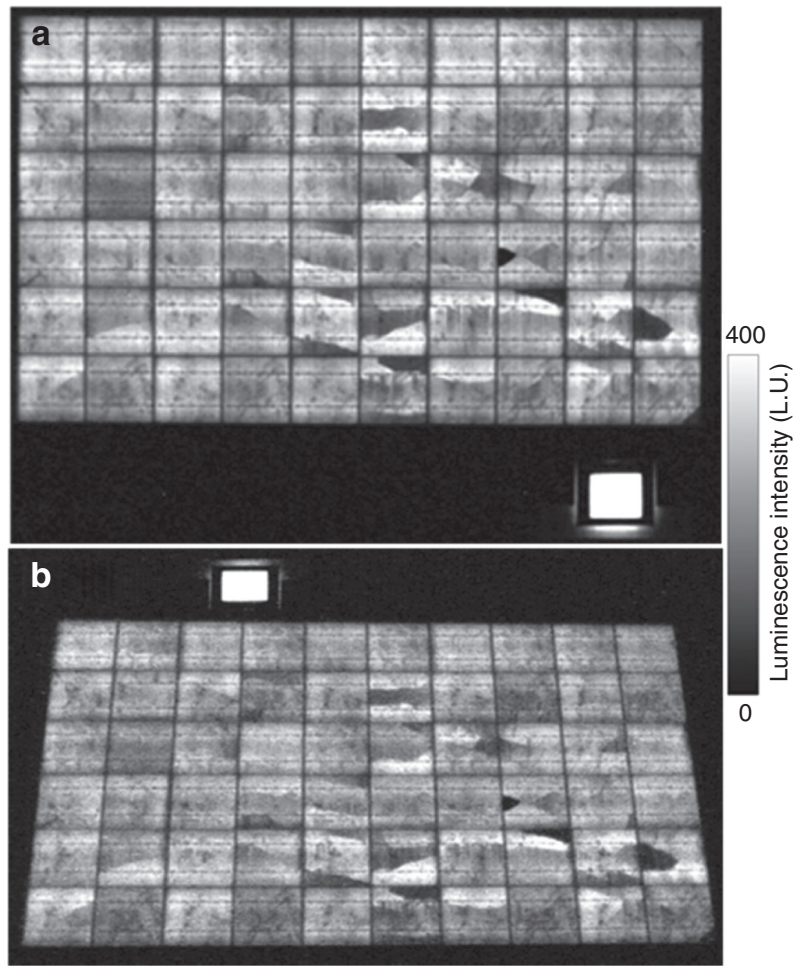

Figure 12: Comparison between indoor (a) and outdoor (b) images. Images were taken with In_C camera for 20 ms integration time.

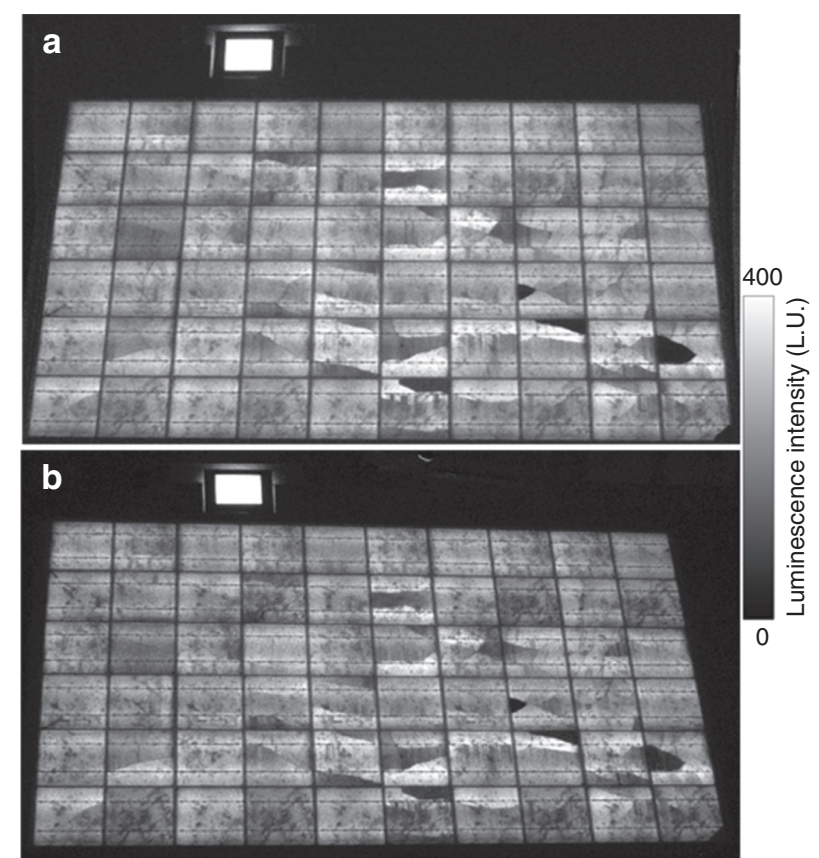

Figure 13: EL image comparison between $60^{\circ}$ angle indoor (a) and $60^{\circ}$ angle outdoor (b) measurements. The images were taken with camera Si_A with 40 s integration time.

Both EL images in Figure 13 are quite similar, as proven by the cumulative histograms in Figure 14, which indicates the robustness of our method even at different perspectives. The small difference at 50 to 250 L.U. might be the influence of the stray light, which increases the intensity level for outdoor measurements.

Further comparison is done in Table 2. Firstly, the slope value of $4.42 \cdot 10^{3}$ from the indoor image is close to $4.06 \cdot 10^{3}$ from the outdoor image. The outdoor image contains more counts with less EL intensity, also proven by the 228 L.U. in comparison with the 249 L.U. in EL mean

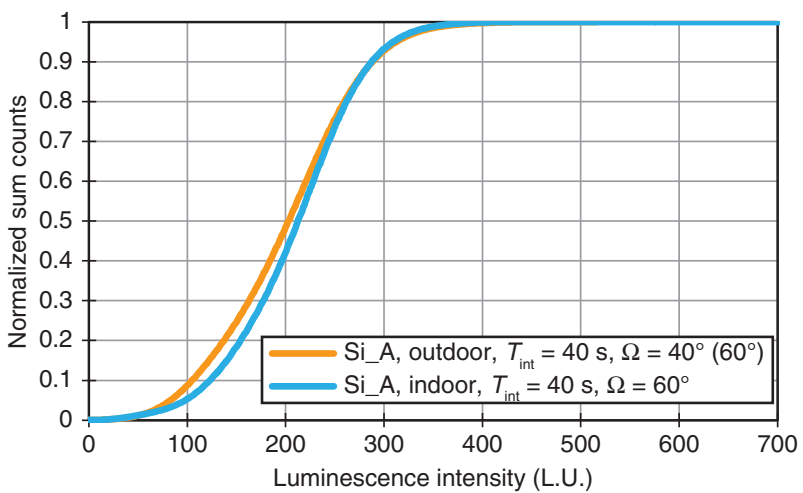

Figure 14: Comparison of Si_A camera's cumulative histograms for indoor and outdoor EL images under $60^{\circ}$ angle. For the outdoor measurements, the module was shifted $40^{\circ}$ and the camera was shifted $20^{\circ}$. 
Table 2: Comparison of the image quality values, SNR, and slope value, for indoor and outdoor measurements. Additionally, the EL mean value and the BS standard deviation are shown.

\begin{tabular}{lrrrr}
\hline & $\begin{array}{r}\text { Slope } \\
\text { value }\end{array}$ & SNR & $\begin{array}{r}\text { EL mean } \\
\text { value (L.U.) }\end{array}$ & $\begin{array}{r}\text { BS std. } \\
\text { dev. }\end{array}$ \\
\hline Indoor & $4.42 \cdot 10^{3}$ & 23.64 & 248.94 & 10.53 \\
Outdoor & $4.06 \cdot 10^{3}$ & 13.24 & 228.09 & 17.23 \\
\hline
\end{tabular}

value from the indoor image. This can be a factor for a variation in the different image resolutions, because of the different perspective and distance for indoor and outdoor acquisition.

Moreover, the difference in temperature between indoor $\left(\sim 25^{\circ} \mathrm{C}\right)$ and outdoor $\left(\sim 5^{\circ} \mathrm{C}\right)$ conditions after 30 min warm-up time still might cause a decrement in the EL intensity (see Fig. 5).

Consequently, the higher noise level in the images might cause an increase of EL intensity in the image, and it leads to a decrement in the SNR. On the other hand, in order to be able to compare and analyse the information, the use of the reference cell to perform the experiment and obtain the data was critical.

\subsection{Black Spot Subtraction}

One major interest in the investigations is whether BS subtraction already enables quantitative comparison of EL images obtained from different sources or if background (BG) subtraction is necessary. For movie recording, the BS subtraction method might increase the throughput. In Figure 15, both EL images are shown.

The background is clearly visible without background subtraction, but the EL signal has the same intensity as it can be seen in Figure 16. Thus, it is sufficient to acquire just one EL image and increase the measurement throughput [15].

\subsection{Quantitative Defect Analysation}

The main finding in our work is that quantitative defect analysis is possible in the same intensity range for all four different cameras. The line scans in Figure 17 indicate that the EL intensity for all cameras is in the same range after the normalisation procedure, which enables quantitative analysis. The higher resolution for the silicon cameras gives more detailed information, especially visible at the bus bar positions with deeper drops at 0.26 and 0.77 . Furthermore, the highest resolution of camera Si_A results in a steeper rise at the right edge at first sight of the inactive area at position 0.65 as compared with camera In_C.

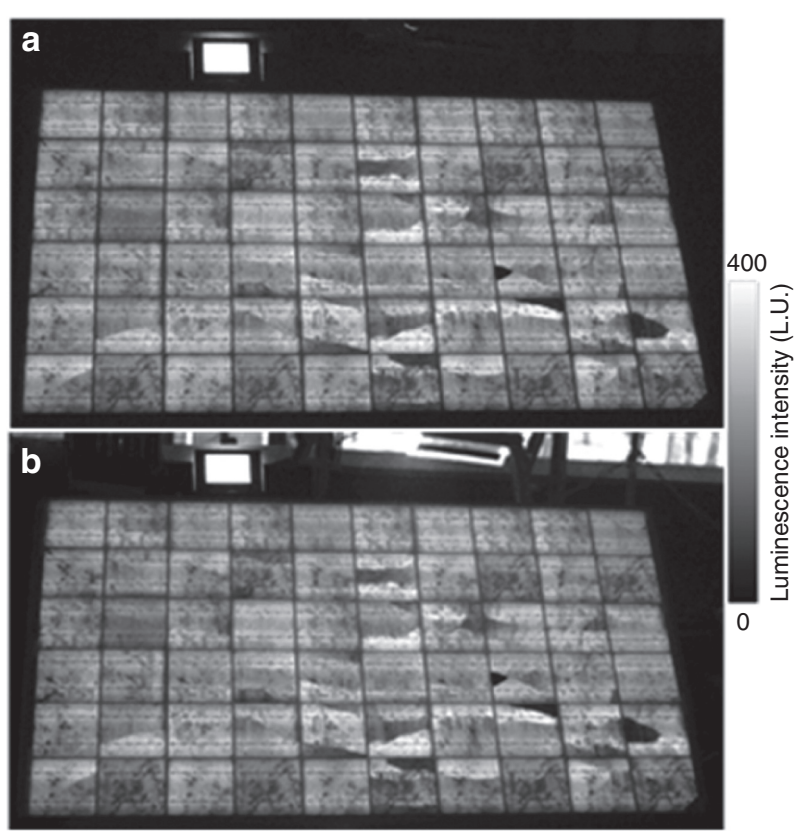

Figure 15: Comparison of the outdoor EL images for camera Si_A with the different subtractions. BG subtraction is shown in (a), and BS subtraction is shown in (b).

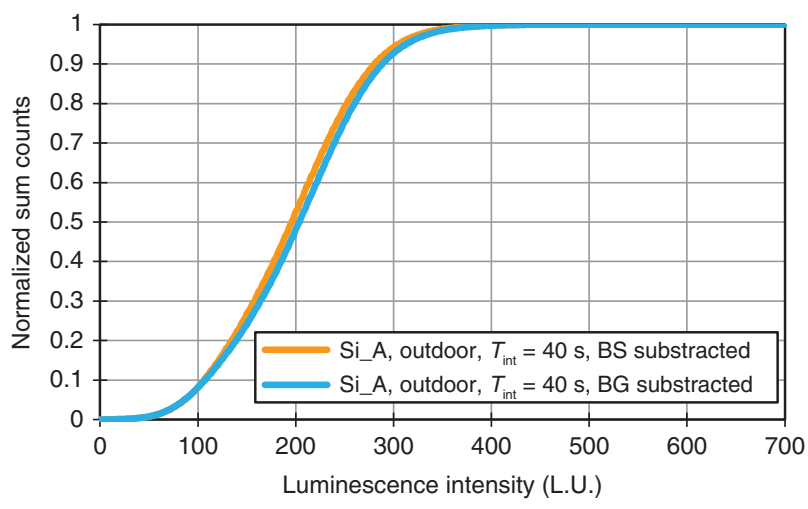

Figure 16: Comparison of the outdoor EL images with cumulative histograms for the different subtractions, BG and BS.

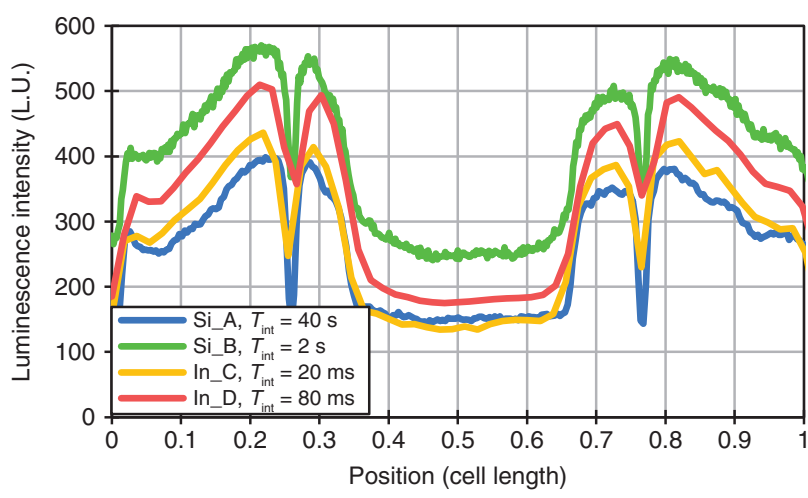

Figure 17: Line scans of the same solar cell with large inactive area in the center between the two busbars, normalised over the cell length for all cameras after the normalisation steps. 


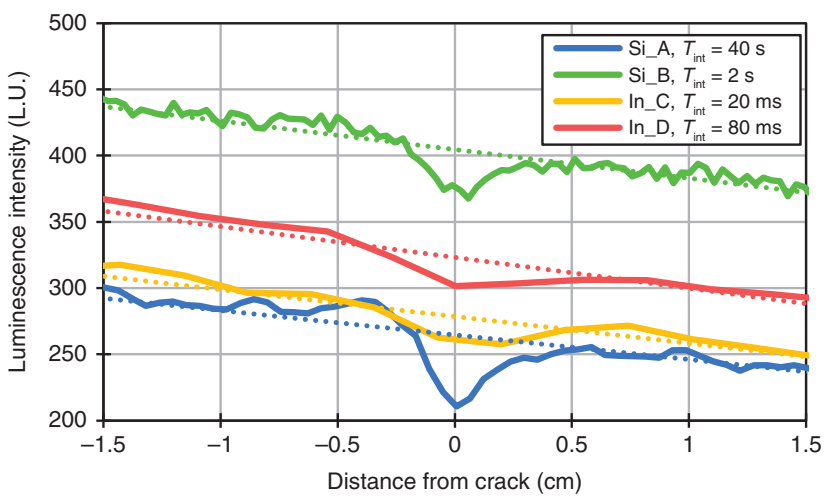

Figure 18: Line scans with trend lines over the same crack centered in the zero point for all cameras after the normalisation steps.

In Figure 18, the line scans over a single crack are shown. The negative deviation from the trend lines indicate the easily detectable crack at position zero. The deviation between crack and trend line can easily be detected by automated defect detection methods. Together with the same intensity range, automated analysis methods can determine energy output and module degradation change for iterated EL measurements $[18,19]$.

\section{Conclusion}

This paper presents, additionally to basic requirements for EL measurements, a robust method to compare EL images obtained from different cameras, with different parameters and different perspectives. We showed that the normalisation with a reference and black spot gives quantitative comparable images for cameras with different detector technologies and materials. The acquired EL images reduce the difference between the detector technologies to the resolution only, which is not surprising because the main advantage for InGaAs detectors is its higher sensitivity and therefore lower integration time, which even enable movie recording.

For movie recording, typically no background subtraction is possible, but with the black spot subtraction, such EL images can be compared with background subtracted ones. Thus, the normalisation method makes highthroughput measurements' methods comparable with nonmoving image recording techniques.

Furthermore, the presented normalisation method can minimise camera influences on the EL images like auto gain, aperture adjustment, or integration time, which are sometimes not reproducible. Thus, the EL images can be analysed only for PV module properties, like parallel or serial resistance for low or high currents. The absolute values can be used to determine grey values classification of inactive areas for the contribution to the cell power output.

All cameras showed different behaviour and have different advantages. The Si detector cameras showed highresolution images, but due to the long integration times, higher than $500 \mathrm{~ms}$ stationary acquisition is necessary, resulting in lower throughput. The low-cost camera Si_B showed the highest noise values, resulting in slightly diffuse images. On the other hand, the InGaAs detector cameras might increase the measurement speed because of their low integration times of about $5 \mathrm{~ms}$. The aim of the camera developers should be the reduction of the camera weight and simultaneously the increase of the mobility in order to use stitching techniques to increase the spatial resolution.

Acknowledgements: This work was supported by the German Federal Ministry for Economic Affairs and Energy (BMWi) for financial funding of this project (AQUAM, Förderkennzeichen 0325807A) and by the Erlangen Graduate School in Advanced Optical Technologies (SAOT) by the German Research Foundation (DFG) in the framework of the German excellence initiative.

\section{References}

[1] A. Luque and S. Hegedus, Photovoltaic Science Handbook of Photovoltaic Science, 2nd ed., John Wiley \& Sons Ltd, Chichester, United Kingdom 2011.

[2] S. T. Thornton and A. Rex, Modern Physics for Scientists and Engineers, 4th ed., Cengage Learning, Brooks/Cole, Boston, MA, USA 2013.

[3] K. Mertens, Photovoltaik: Lehrbuch Zu Grundlagen, Technologie Und Praxis, 3th ed., Carl Hanser Verlag GmbH, Munich, Germany 2015.

[4] M. A. Green, J. Zhao, A. Wang, P. J. Reece, and M. Gal, Nature 412, 805 (2001).

[5] T. Fuyuki, H. Kondo, Y. Kaji, A. Ogane, and Y. Takahashi, J. Appl. Phys. 101, 023711-1, (2007).

[6] U. Rau, Phys. Rev. B 76, 085303 (2007).

[7] M. Köntges, S. Kajari-Schröder, I. Kunze, and U. Jahn, in: 26th European Photovoltaic Solar Energy Conference and Exhibition, Eionet, Hamburg, Germany 2011, p. 3290.

[8] C. Buerhop, S. Wirsching, A. Bemm, T. Pickel, P. Hohmann, et al., Prog. Photovoltaics Res. Appl. 26, 261 (2018).

[9] O. Breitenstein, A. Khanna, Y. Augarten, J. Bauer, and K. Iwig, Phys. Status Solidi 9, 7 (2010).

[10] K. G. Bedrich and T. R. Betts, in: 12th Photovoltaic Science, Applications and Technology Conference (PVSAT-12), University of Liverpool, Liverpool, UK 2016. 
[11] K. G. Bedrich and T. R. Betts, in: 2016 IEEE 43rd Photovoltaic Specialists Conference (PVSC), IEEE, Portland, OR, USA 2016, p. 1532.

[12] J. Colvin, in: 35th International Symposium on Test Failure and Analysis, ASM, Russell Township, OH, USA 2009.

[13] B. E. A. Saleh and M. C. Teich, Photons in Semiconductors, in Fundam. Photonics, vol. 5, John Wiley \& Sons, Inc., Hoboken NJ, USA 1991.

[14] T. Kirchartz, V. Huhn, A. Gerber, B. E. Pieters, and U. Rau, Advanced Characterization Techniques for Thin FilmSolar Cells, 2nd ed., in: Electroluminescence Analysis of Solar Cells and SolarModules, Chapter 3, Wiley-VCH Verlag GmbH \& Co. KGaA, Weinheim, Germany 2016, p. 71.
[15] J. Adams, B. Doll, C. Buerhop, T. Pickel, J. Teubner, et al. in 32nd Eur. Photovolt. Sol. Energy Conf. Exhib. (2016).

[16] C. Boncelet, Chapter 7 - Image Noise Models, 2nd ed., in Essential Guide to Image Process. 7, 2nd ed., Elsevier, the Netherlands 2009.

[17] A. Rose, Vision: Human and Electronic, Springer, New York, NY, USA 1973.

[18] M. Köntges, M. Siebert, D. Hinken, U. Eitner, K. Bothe, and T. Potthof, in: 24th European Photovoltaic Solar Energy Conference, Hamburg, Germany 2009, p. 3226.

[19] S. Deitsch, C. Buerhop-lutz, A. Maier, F. Gallwitz, and C. Riess, Segmentation of Photovoltaic Module Cells in Electroluminescence Images, arXiv (2018). DOI: 10.1016/j.solener.2019. 02.067 . 